\title{
Acute Necrotizing Encephalitis as a Probable Association of COVID-19
}

\author{
Neeraj Kumar ${ }^{1}$, Subhash Kumar ${ }^{2}$, Abhyuday Kumar ${ }^{3}$, Binod K Pati ${ }^{4}$, Amarjeet Kumar ${ }^{5}$, Chandramani Singh ${ }^{6}$, Asim Sarfraz ${ }^{7}$
}

\begin{abstract}
Background: Meanwhile, over 50 lakh people have now been affected by coronavirus disease 2019 (COVID-19) across the globe. There are various reports on neurological manifestations of COVID-19, which have attracted broad attention. Acute necrotizing encephalopathy (ANE) is a rare complication of influenza and other viral infections and has been related to intracranial cytokine storm, which results in breach in blood-brain barrier leading to encephalitis like presentation. We report an unusual case of acute necrotizing encephalitis as a solitary presentation of COVID-19. Case description: We report a case of 35-year-old man from Bihar, presented to our emergency department in unconscious state, with highgrade fever and vomiting since last 5 days. Previous magnetic resonance imaging (MRI) brain showed a left parasellar-middle cranial fossa mass looks most likely like an invasive meningioma. Urgent non contrast computed tomography scan (NCCT) brain showed that mass as well as hypodensities in both thalami and left caudate nucleus. As per our institutional protocol, clinical management of raised intracranial pressure was initiated. As there is no current evidence from any randomized control trails (RCTs) to recommend any specific treatment for suspected or confirmed patients with COVID-19 with acute necrotizing encephalitis.

Conclusion: Our case highlights the importance of identifying encephalitis as a presenting sign of COVID-19 based on NCCT findings with normal cerebrospinal fluid (CSF) and normal chest X-ray (CXR) findings.

Keywords: Acute necrotizing encephalitis, Altered sensorium, Computed tomography, COVID case report, Coronavirus disease 2019 in India, Encephalopathy, Magnetic resonance imaging.

Indian Journal of Critical Care Medicine (2020): 10.5005/jp-journals-10071-23636
\end{abstract}

\section{INTRODUCTION}

Coronavirus disease 2019 (COVID-19) is an infectious disease caused by severe acute respiratory syndrome coronavirus 2 (SARS-CoV-2), previously known as 2019 novel coronavirus (2019-nCoV), a strain of coronavirus. The first cases were seen in Wuhan, China, in December 2019, before spreading globally, ${ }^{1}$ and officially recognized as a pandemic on March 11, 2020.2

In India, the number of COVID-19 patients has surpassed 300,000 and is increasing exponentially. Meanwhile, over 50 lakh people have now been affected by COVID-19 across the globe. Around 69\% coronavirus patients in India who were tested did not show any symptoms of the infection as per Indian Council of Medical Research (ICMR) leading to an increased evidence of "silent spreaders" who are unknowingly infecting others.

Acute necrotizing encephalopathy (ANE) is a rare complication of influenza and other viral infections and has been related to intracranial cytokine storm, which results in blood-brain barrier breakdown, but without direct viral invasion or parainfectious demyelination. ${ }^{3}$ Accumulating evidence suggests that a subgroup of patients with severe COVID-19 might have a cytokine storm syndrome. 4

\section{Case Description}

A 35-year-old man from Bihar, presented to our emergency department in unconscious state, with high-grade fever and vomiting since last 5 days. His relatives gave the history of headache for last 6 months. For his persistent headache, a magnetic resonance imaging (MRI) of brain had been done at an outside hospital 10 days earlier, which showed a left parasellar-middle cranial fossa mass extending via the foramen ovale into the left infratemporal fossa and its dural based and looks most likely like

\footnotetext{
1,5 Department of Trauma and Emergency, All India Institute of Medical Sciences, Patna, Bihar, India

${ }^{2}$ Department of Radiodiagnosis, All India Institute of Medical Sciences, Patna, Bihar, India

${ }^{3}$ Department of Anaesthesiology, All India Institute of Medical Sciences, Patna, Bihar, India

${ }^{4,7}$ Department of Microbiology, All India Institute of Medical Sciences, Patna, Bihar, India

${ }^{6}$ Department of Community and Family Medicine, All India Institute of Medical Sciences, Patna, Bihar, India

Corresponding Author: Neeraj Kumar, Department of Trauma and Emergency, All India Institute of Medical Sciences, Patna, Bihar, India, Phone: +91 8210104972, e-mail: neeraj.jnmc@gmail.com

How to cite this article: Kumar N, Kumar S, Kumar A, Pati BK, Kumar A, Singh $C$, et al. Acute Necrotizing Encephalitis as a Probable Association of COVID-19. Indian J Crit Care Med 2020;24(10):991-994.

Source of support: Nil

Conflict of interest: None
}

an invasive meningioma (Fig. 1). Patient had no recent history of travel or contact with other COVID-19 patients. On examination, Glasgow coma scale (GCS) was $\mathrm{E}_{1} \mathrm{~V}_{1} \mathrm{M}_{2}$, chest auscultation showed normal vesicular breath sounds, heart rate (HR) 127/minute, blood pressure (BP) $138 / 87 \mathrm{~mm} \mathrm{Hg}$, temperature $104^{\circ} \mathrm{F}$, respiratory rate $36 /$ minute, and $\mathrm{SPO}_{2}$ was $95 \%$ on room air. A plain computerized tomography (CT) scan of the head under our institutional COVID19 protocol was immediately performed, which showed the mass as well as hypodensities in both thalami and left caudate nucleus (Fig. 2). According to Modified Early Warning Score, ${ }^{5}$ this patient was classified as suspected COVID-19 severe case with a score of 

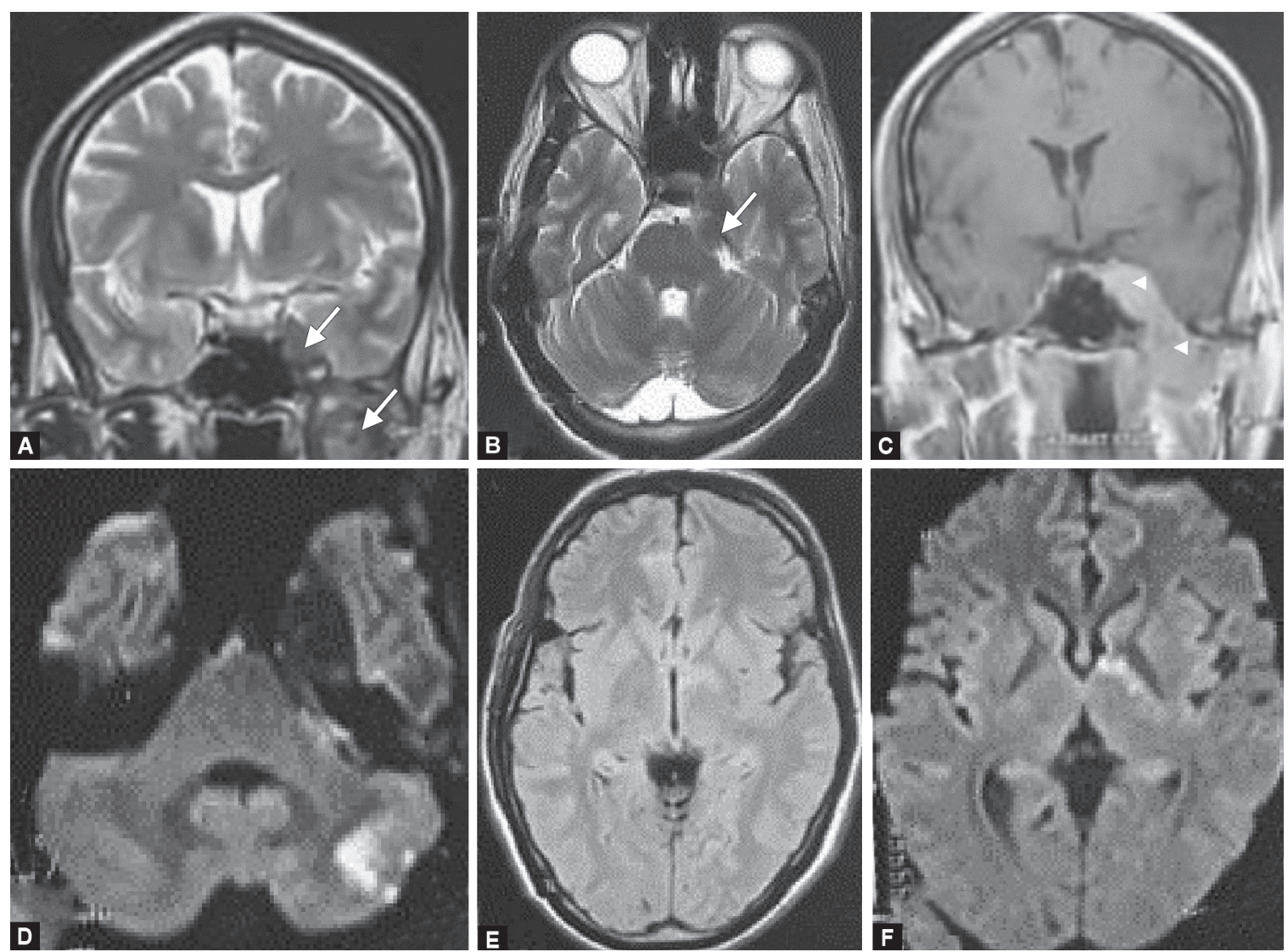

Figs $1 \mathrm{~A}$ to $\mathrm{F}$ : Plain and contrast-enhanced MRI scan 10 days prior to admission at another center outside our institute: (A) Coronal T2W image through sella showing a hypointense mass in the left parasellar region, extending into the left infratemporal fossa (thin short arrows); (B) Axial T2W image at level of superior cerebellar peduncles showing the mass has extended across the tentorium into the posterior fossa and is indenting the brainstem (thin dashed arrow); (C) Coronal post-contrast T1W image showing diffuse enhancement of the mass (white small arrowheads); (D) Axial diffusion-weighted image (DWI) showing lack of restriction in the mass, irregular diffusion restricting lesions are seen in left hemicerebellum; (E) Axial FLAIR image at level of third ventricle showing subtle hyperintensity in the left anterior thalami, globus pallidus region; (F) Axial DWI showing tiny hyperintensities at the left anterior thalamus-globus pallidus region corresponding to the hyperintense area in the FLAIR image

12/21 points and was admitted in a dedicated COVID-19 intensive care unit (ICU). Chest radiographs on the day of admission and next day in the isolation were within the normal limits (Fig. 3). In view of airway protection and poor GCS, trachea was intubated with endotracheal tube of size $8 \mathrm{~mm}$ by wearing complete personal protective equipment (PPE) zone III protection gear and patient was put on synchronized intermittent mandatory ventilation (SIMV) mode of mechanical ventilation. As per Centers for Disease Control and Prevention (CDC) recommendations, nasopharyngeal swab was collected under recommended biosafety precaution. It was placed in viral transport medium (VTM) and sent to laboratory maintaining cold chain. CDC/WHO recommended real-time PCR was performed and ORF1b gene of SARS-CoV-2 was detected in this patient. On the day of admission, cerebrospinal fluid analysis showed increased protein and no bacterial growth, and tests for herpes simplex virus 1 and 2, and varicella zoster virus were also negative. The various laboratory parameters like $\mathrm{Hb} \%$ of $14.4 \mathrm{~g} / \mathrm{dL}$, total leucocyte count (TLC) of $14.81 \times 10^{9} / \mathrm{mm}^{3}$, neutrophils $85 \%$, lymphocytes $10 \%$, and other parameters were within normal limit. His arterial blood gas (ABG) was $\mathrm{pH}: 7.51, \mathrm{PaO}_{2}: 131, \mathrm{PaCO}_{2}: 22, \mathrm{HCO}_{3}$ : 18, lactate: 1.3. Patient was managed with hydroxychloroquine $400 \mathrm{mg}$ twice daily, azithromycin $500 \mathrm{mg}$ once daily, and injection amoxicillin-clavulanic acid $1.2 \mathrm{~g}$ intravenous thrice daily, intravenous methyl prednisolone $1 \mathrm{~g}$ once daily, along with other standard ICU therapy. On second day, patient started showing features of raised intracranial pressure like increased BP, bradycardia, and papilledema. Patient was managed with propofol infusion and mannitol. An MRI brain was planned; however, patient had cardiopulmonary arrest and expired next day after about 36 hours of admission.

\section{Discussion}

We report the first presumptive case of ANE as a solitary presentation of COVID-19 from India. As in literature, this COVID-19 virus can affect multiple organs including the nervous system where the receptor is predominantly expressed by neurons. This COVID-19 virus is believed to reach the central nervous system (CNS) via two major routes. After infecting the nasal mucosa, coronaviruses can invade the brain through the cribriform plate, advancing along the olfactory nerve. Alternatively, coronaviruses can reach the capillaries via the bloodstream and may interact with angiotensin converting enzyme-2 (ACE2) to invade and replicate within the endothelium, leading to endothelial damage which allows the greater viral access into the neural tissues. ${ }^{6}$ Various neurological manifestations can be seen in viral infections, including meningitis, meningoencephalitis, encephalitis, acute demyelinating encephalomyelitis with or without hemorrhage, demyelinating polyneuropathy, transverse myelitis, and the imaging findings vary according to the condition. 

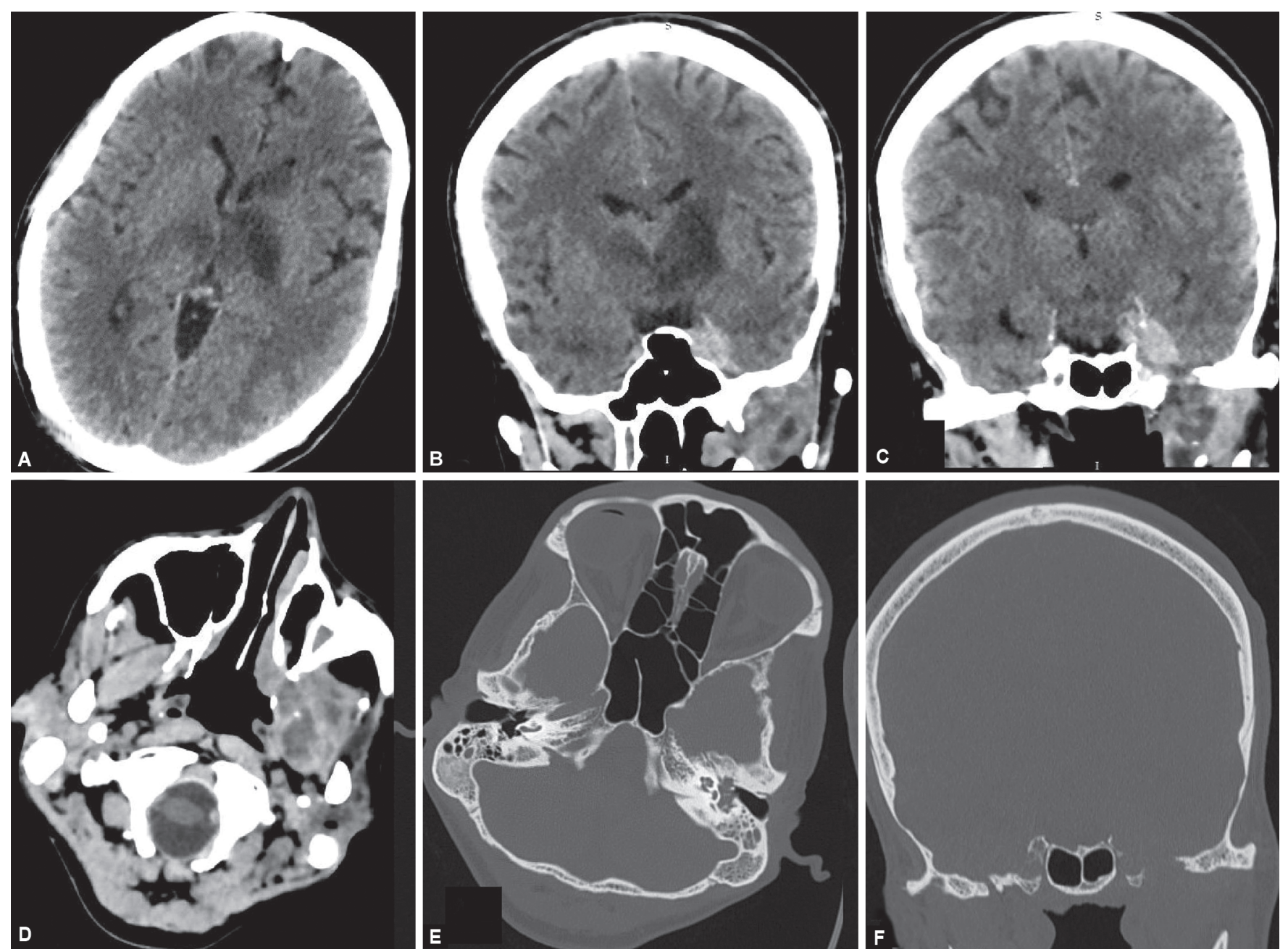

Figs 2A to F: CT head at admission: (A) Axial images showing hypodensities in bilateral thalami and left basal ganglia; (B and C) Coronal reformatted images showing the hypodensities; the left parasellar mass has homogeneous hyperdense appearance with extends through the left foramen ovale; (D) Axial image at nasopharyngeal level showing the infratemporal mass to be heterogeneous with necrotic areas, note similar changes in the left carotid also; (D) Axial image, bone window showing left otomastoiditis and scalloping of the left petrous bone by the mass; (E) Coronal reformatted image, bone window showing the widened left foramen ovale; (F) Bone window showing the widened left foramen ovale

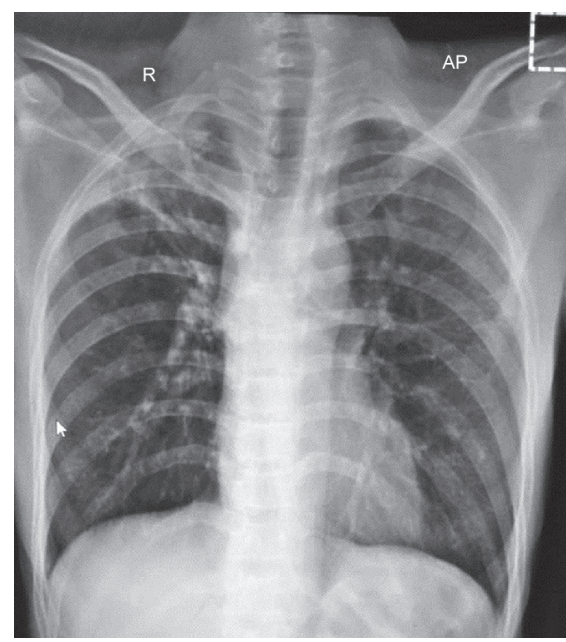

Fig. 3: A normal chest radiographs on the day of admission and next day in the isolation intensive care unit (ICU)
Acute necrotizing encephalopathy has a classic radiological pattern with involvement of both thalami similar to our case. Acute necrotizing encephalopathy has been associated with cytokine storms and can be life-threatening. Neurological symptoms were the sole presentation in our case and were having radiological features of ANE without the involvement of any other system. Various neurological symptoms seen in COVID-19 infection can manifest as acute stroke (6\%), consciousness impairment (15\%), and skeletal muscle injury (19\%). ${ }^{7}$ In our case, there was an area of signal change in left cerebellum also, seen in the MRI done 10 days earlier, which could be ischemic. As per literature, both central and peripheral nervous systems are affected following SARS virus. These viruses may be detected in cerebrospinal fluid (CSF) by reverse transcriptase-polymerase chain reaction (RT-PCR) but not almost always as these viruses can be cultured from brain tissue also as seen in patient presented with acute encephalopathy with seizures as per literature. ${ }^{8}$

There is no current evidence from any randomized control trails (RCTs) to recommend any specific treatment for suspected 
or confirmed patients with COVID-19 with acute necrotizing encephalitis. No specific antivirals are recommended for the treatment of COVID-19 due to lack of adequate evidence from literature. High-dose corticosteroids, immunoglobulins, and plasmapheresis can be tried as treatment options could potentially be useful for patients with ANE if diagnosed early.

\section{Conclusion}

Our case highlights the importance of identifying encephalitis as a presenting sign of COVID-19 based on non contrast computed tomography scan (NCCT) findings with normal CSF and normal chest X-ray (CXR) findings. Intensivists should be aware of this presentation among COVID-19 patients presenting with altered mental status.

\section{References}

1. "A Novel Coronavirus from Patients with Pneumonia in China, 2019 | NEJM.".

2. "WHO Director-General's Opening Remarks at the Media Briefing on COVID-19 - 11 March 2020.".
3. Rossi A. Imaging of acute disseminated encephalomyelitis. Neuroimaging Clinics 2008;18(1):149-161. DOI: 10.1016/j. nic.2007.12.007.

4. Mehta P, McAuley DF, Brown M, Sanchez E, Tattersall RS, Manson $\mathrm{JJ}$, et al. COVID-19: consider cytokine storm syndromes and immunosuppression. Lancet 2020;395(10229):1033-1034. DOI: 10.1016/S0140-6736(20)30628-0.

5. Liao $X$, Wang $B$, Kang $Y$. Novel coronavirus infection during the 2019-2020 epidemic: preparing intensive care units-the experience in Sichuan Province, China. Intensive Care Med. 2020;46(2):357-360. DOI: 10.1007/s00134-020-05954-2.

6. Wu Y, Xu X, Chen Z, Duan J, Hashimoto K, Yang L, et al. Nervous system involvement after infection with COVID19 and other coronaviruses. Brain Behav. Immun. 2020;87:18-22. DOI: 10.1016/j.bbi.2020. 03.031.

7. Neurological manifestations of hospitalized patients with COVID-19 in Wuhan, China: a retrospective case series study. (2020).Accessed: March 21, 2020: https://www.medrxiv.org/content/10.1101/2020.02. 22.20026500v1.

8. Ellul MA, Benjamin L, Singh B, Lant S, Michael BD, Easton A, et al. Neurological associations of COVID-19. Lancet Neurol 2020;19(9):P767-P783. DOI: 10.1016/S1474-4422(20)30221-0. 